\title{
Neurociencia, neurología, y psiquiatría: Un encuentro inevitable ${ }^{1}$
}

RESUMEN: El extraordinario desarrollo de la neurobiología, psicobiología y neuropsiquiatría a partir de la segunda mitad del s. XIX plantea hoy un reto: reconstruir y quizá reelaborar una epistemología de la mente y el cerebro que abarque ya desde los genes a la subjetividad individual.

PALABRAS CLAVE: Mente-cerebro, Neurobiología, Psicobiología

\begin{abstract}
The extraordinary progress of neurobiology, psychobiology and neuropsychiatry, unfolding since the second half of the XIX century, offers us a challenge today: to reconstruct -perhaps re-elaborate- an epistemology of mind and brain that encompasses from genes to individual subjectivity.
\end{abstract}

KEY WORDS: Mind-brain, Neurobiology, Psychobiology

Natura abhorret vacuum, tamquam humana mens abhorret incertitudinem.

(Anónimo)

Begin with certainties, and you shall end in doubts; but if you will be content to begin with doubts, you will end in certainties.

(Francis Bacon)

Poca discrepancia puede suscitar la afirmación de que la convergencia producida entre las diversas disciplinas que dieron lugar a la aparición de la Neurociencia en los años 60 ha producido avances espectaculares en el conocimiento de la biología del sistema nervioso y de las relaciones de este con la conducta. Pero los jalones establecidos por la Neurociencia ¿han servido para orientar las investigaciones en Neurología y Psiquiatría, y a incrementar sus relaciones con la propia Neurociencia?

Narra en uno de sus Apólogos Luis Martín Santos (1) la historia de los exploradores españoles que para orientarse en las inmensas planicies de América plantaban hileras de estacas, con las que jalonaban rutas y marcaban orientaciones para proseguir su exploración de lo desconocido, deshacer con facilidad caminos erróneos, y no acometer engañosas marchas en círculo o espiral. En la exploración del cerebro se han trazado multitud de mapas, nosologías y marcos conceptuales a partir de abordajes metodológicos diversos, y desde la clínica, la psicología y la epistemología se han

\footnotetext{
${ }^{1}$ Texto de la conferencia con el mismo título dada por el Dr. Carlos Avendaño en las XVIII Jornadas de la Asociación Española de Neuropsiquiatría - Huelva, 4-6 de abril, 2002.
}

Rev. Asoc. Esp. Neuropsiq., 2002, vol XXII, n. o 83, pp. 65-89 
propuesto sucesivos y a veces enfrentados marcos heurísticos para entender y abordar la mente. Parece justificable ceder a la tentación de enmarcar, jalonar y limitar lo inmenso y desconocido. Con ello hemos avanzado y razonablemente esperamos conquistar el cerebro como los exploradores conquistaron América. Pero Martín Santos nos recuerda que la sabia estrategia de aquellos exploradores permitió con frecuencia a tribus caníbales seguir las rutas marcadas para dar buena cuenta de los confiados intrusos. La búsqueda excesiva de seguridad y de certidumbre pueden ser malas consejeras.

\section{De lo unido a lo disperso}

La mente humana ha observado el cerebro y ha reflexionado sobre sí misma casi al unísono, desde que en el s. V a.C. se desarrolló la teoría de los 4 elementos (Empédocles de Agrigento, 490-430 a.C.) y éstos se asociaron a los 4 humores en el Corpus Hippocraticum (Hipócrates de Cos, 460-370 a.C.), se realizaron las primeras disecciones anatómicas del cerebro registradas (Alcmeon de Crotona, de las que deduciría que el cerebro era el órgano central de las sensaciones), y uno de los últimos presocráticos, Anaxágoras de Klazomene (500-428 a.C.) propuso que el cerebro era el órgano de la mente.

Aunque no es éste momento, ni soy la persona adecuada, para explayarse en análisis históricos, intentaré entresacar algunos hechos e ideas de fuentes bien acreditadas (2-6) en apoyo de un argumento inicial, que la reflexión y el estudio de lo cerebral y lo mental han estado mayoritariamente unidos hasta fechas my cercanas a nuestros días.

Vesalio (1514-1564), médico y máximo exponente de la Anatomía renacentista, en su abierto cuestionamiento de las posiciones dominantes ya milenarias de Galeno, realizó disecciones y descripciones anatómicas extraordinarias del cerebro, urgió a sus contemporáneos a reexaminar la estructura y función del cerebro con una mente abierta, y se lamentó de que la sola anatomía no pudiera permitirle comprender cómo el cerebro regula la imaginación, el razonamiento y la memoria (componentes básicos del intelecto según Galeno). Su De Humani Corporis Fabrica quiso enmendar las confusiones entre forma y función de Galeno manteniendo la descripción del cuerpo humano en el terreno de la primera, sin avanzar un paso en la segunda. No es de extrañar esa aprensión, pues aún seguía plenamente vigente una fusión sustancial entre la medicina y la filosofía. Habría que esperar un cuarto de siglo tras la muerte de Galeno a que Felix Platter (1536-1614), médico y anatomista de Basilea separara la medicina de la filosofía (7) para adscribirla a la ciencia natural; minucioso observador de síntomas, causas y tratamientos, fue pionero en el estudio de la conducta y la neuropatología de los enfermos mentales. 
Algo más de un siglo después de la Fabrica (1543) se publicaría el Cerebri anatome (1664) de Thomas Willis (1621-1675), de gran influencia en los siglos XVII y XVIII. Médico como Vesalio, y también como él asentado aún en la teoría galénica de la producción y el flujo del spiritus animalis en el cerebro (para Galeno se produciría en los ventrículos y fluiría hacia el parénquima y los nervios), Willis consagró al cerebro como "el origen y fuente de todos los movimientos y concepciones", y aportó la primera propuesta moderna de localización de funciones cerebrales: la imaginación se asentaría en el cuerpo calloso (con el centro oval inmediato), por ser casi constante entre individuos, mientras que la memoria y la voluntad se asentarían en las circunvoluciones cerebrales. Además introdujo el término Neurología como 'doctrina de los nervios'.

Sólo 2 años antes de la aparición del libro de Willis se publicó, póstumo, De Homine de René Descartes (1596-1650). Los conocimientos físicos y anatómicos acumulados demandaban ya explicaciones más extensas para el flujo del spiritus animalis y la relación entre el alma y el órgano en el que ésta parecía definitivamente asentarse. Y Descartes, desde la duda como método para eliminar el error al que inducen los sentidos, elaboró la primera teoría completa moderna sobre el cerebro y la mente, proponiendo una ingeniosa explicación mecanicista al flujo de los spiritus hacia y desde el cerebro, y dejando a la mente, lo pensante, en un plano sustancial diferente de todo lo sensible, lo extenso.

Las consecuencias de esta dualismo cartesiano se siguen sintiendo hoy. Ciertamente consagró un problema ontológico sobre la mente y el cerebro que se extiende hasta hoy, pero también liberó a la emergente ciencia moderna de la necesidad de referenciar sus avances a lo religioso-filosófico: las manipulaciones astronómicas de Galileo (1564-1642) se referirían así a res extensa, plenamente abordable por la razón. Las teorías aplicadas a la naturaleza dejaron de ser derivaciones necesarias de apriorismos ontológicos y metafísicos a los que había que conformar los hechos observables, para pasar a convertirse en fundamentos del método científico actual.

Opuesto al racionalismo en muchos aspectos, el empirismo que se desarrolló en Inglaterra desde fines del s. XVI a partir de Francis Bacon (1561-1626) y Thomas Hobbes (1588-1679) aportó el uso de la inducción, el recurso a la experiencia y una progresiva suspicacia sobre la capacidad de la razón para ofrecer conocimiento sobre la realidad de las cosas. De hecho, no existirían ideas innatas ni inconscientes: las ideas simples procedentes de la percepción darían lugar a las ideas -funciones mentalescomplejas, a travéés de la reflexión y un proceso de asociación de ideas (John Locke, 1632-1704). Ya en pleno s. XVIII, David Hume (1711-1776), niega la posibilidad del conocimiento de las cosas, que sólo causarían impresiones en nuestra mente, e impone el escepticismo en metafísica -no hay causas, solo coexistencia o sucesión de impresiones o ideas.

Racionalismo y empirismo en desarrollo expandieron en la Ilustración el método científico y abrieron un abanico de interpretaciones sobre qué es el hombre, desde el materialismo mecanicista radical del médico LaMettrie (1709-1751) y el filósofo 
D’Holbach (1723-1789) -para quienes sólo diferencias de grado separarían el mecanismo animal del humano, y la mente sería un producto material del cerebro-, hasta diversas formas de vitalismo, más pasivo o reactivo en Albrecht von Haller (1708-1777), más productivo y espontáneo en otros como Wolff. En ese período se produjo una multiplicación de observaciones y estudios empíricos sobre el sistema nervioso: en el s. XVIII se descubren "centros" y "vías" funcionalmente específicas: el centro respiratorio en el bulbo (Legallois), el papel sensitivo o motor de raíces dorsales y ventrales (Charles Bell y François Magendie), el cruzamiento de las pirámides y su relación con trastornos motores cruzados tras lesiones cerebrales (Domenico Mistichelli, Prof. de Medicina en Pisa y Pourfour du Petit, médico y cirujano militar), etc.

El idealismo alemán iniciado por Kant (1724-1804) intenta conseguir una síntesis entre racionalismo y empirismo, pero se ocupa poco de la naturaleza y mucho de la filosofía, que entra en una profunda crisis tras la muerte de Hegel (1770-1831). En el seno de esa crisis nació el positivismo con Auguste Comte (1789-1877), curiosa mezcla de teoría del conocimiento y de la ciencia con una religiosidad materialista y una ética social. El positivismo consagra la voluntad de aceptar la realidad como hechos sensibles, sin esquemas apriorísticos superpuestos, renunciando a todo lo que no sean datos, hechos y leyes. Estos son la base del conocimiento, el cual, no obstante, es siempre relativo a la realidad histórico-social del individuo. La importancia del positivismo en la ciencia de nuestros días es innegable. Propició la fe en la 'religión de los hechos' que diría Cajal, y la expansión del reduccionismo, que ha sido fundamental para el crecimiento del conocimiento científico.

En ese contexto mayoritariamente positivista, con un explosivo desarrollo de la física y la química, sobre el fisionomismo en boga desde el último tercio del s. XVIII, la demostración de la electricidad animal desde la publicación de Galvani en 1791 y con la reciente aparición de la frenología se produce con el cambio de siglo un extraordinario auge de los estudios sobre el cerebro y la mente. No sorprendentemente, buena parte de este auge se nutrió de encendidos enfrentamientos entre concepciones contrapuestas acerca de ambas entidades. Veamos de forma muy somera los hitos más destacados que jalonaron el siglo XIX [cf. algunas referencias adicionales de interés: (7-16)].

- Ácida pugna entre el localizacionismo frenológico de Franz Joseph Gall 1758-1828 y Johann Spurzheim (1776-1832) -difusor exitoso entre los médicos clínicos de la frenología- y la equipotencialidad cortical, principalmente liderada por MarieJean-Pierre Flourens (1794-1867), fisiólogo que utilizó ampliamente el método de lesiones experimentales en anfibios, aves y mamíferos. La poca fundamentación metodológica y neuropatológica de la frenología, y la aparentemente sólida fundamentación experimental -y en parte clinicopatológica, sobre casos de hidrocefalia- de la equipotencialidad cortical dejarían a ésta como doctrina dominante hasta la presentación del caso Mr. Leborgne ("Tan”) por Broca en 1861.

- Proposición de la teoría celular Mathias Schleiden (1804-1881) y Theodor Schwann (1810-1882), publicada en 1839, un año después de que Jan Evangelista Purkinje 
(1787-1869) publicara 1os dibujos de sus células del cerebelo. Aunque rápidamente se aceptó la estequiología celular para todo el organismo, sin embargo durante el resto del s. XIX se mantuvo la polémica sobre la existencia de células individuadas en el SNC, al no poderse determinar si sus prolongaciones formaban o no una red continua.

- La psiquiatría basada en la teoría moral de la enfermedad mental dominó a principios del s. XIX con Philippe Pinel (1745-1826, primero en quitar las cadenas a los enfermos mentales en la Salpêtrière, e introductor del término demencia en 1797) y su seguidor Jean Etienne Dominique Esquirol (1782-1840). Contra esa posición se desarrollaría en la mitad del siglo una reacción fisicalista, la psiquiatría alienista que, descartada la capacidad explicativa o terapéutica de la frenología, buscaba anomalías en el cerebro que explicaran la patología. Theodor Meynert (1833-1893), psiquiatra y anatomista, llevó al extremo del determinismo neuropatológico sus interpretaciones patofisiológicas psiquiátricas en Psiquiatría: Enfermedades del Prosencéfalo (1874), lo que probablemente contribuyó sin quererlo al declinar de fines de siglo de la Neuropsiquiatría.

- La publicación de El origen de las especies en 1859 produjo un salto cualitativo en la ya extendida idea del transformismo o evolución de las formas vivas. Charles Darwin (1809-1882) añadió a la idea de que todas las especies proceden de la paulatina transformación de las anteriores una causa: la selección natural y la supervivencia en ella de los más aptos. Durante más de una década Darwin dejó a un lado la aplicación de su teoría al caso del hombre, quizá influido -además de por la presión cultural y religiosa dominante- por la negativa de Alfred Russell Wallace a aceptar que la selección natural pudiera actuar igualmente sobre el hombre, pues no se podrían explicar así las propiedades más complejas de la mente humana. Pero en sus obras ulteriores, El linaje del hombre (1871) y La expresión de las emociones en el hombre y los animales (1872) aceptó también este extremo.

- La asociación de afasia con lesión focal en el lóbulo frontal convirtió a Broca al localizacionismo, pero también fue un golpe de gracia contra la frenología: existía una localización de funciones corticales, pero no una correlación con los accidentes óseos. Este acontecimiento tuvo una gran repercusión, no sólo por el detallado estudio que presentó del paciente y su lesión cerebral, sino además por la respetada personalidad de Broca, médico y científico prestigioso. Sólo 9 años después (1870) se descubre la corteza motora y su mapa somatotópico en el perro mediante estimulación eléctrica cortical de baja intensidad por Gustav Fritsch 1838-1927, médico y anatomista, y Eduard Hitzig (1838-1907), psiquiatra, que invitó a su colega a realizar el experimento en su casa en Berlín. Entre 1863 y 1870 John Hughlings Jackson (1835-1911) describió sus observaciones sobre centenares de casos de epilepsias parciales y hemiplejias y, sobre todo, teorizó sobre la organización somatotópica de una representación motora -más que un locus motor anatómicamente concreto- en la corteza, que ejecutaría los actos motores voluntarios -los involuntarios y automáticos dependerían de estructuras subcorticales. 
- La aparición de métodos nuevos y por primera vez realmente eficaces de fijación, corte y tinción de tejido cerebral, y especialmente el método de Golgi, produjeron avances revolucionarios en el conocimiento de la estructura histológica y celular del sistema nervioso, y la confirmación progresiva de la teoría neuronal, postulada por Wilhelm His (1831-1904) y Rudolph Albert von Kölliker (1817-1905), demostrada por vez primera con datos convincentes en 1887 por August Forel (1848-1931) y Fridtjof Nansen (1861-1930), confirmada inapelablemente por la ingente obra de Santiago Ramón y Cajal (1852-1934) a partir de 1888, y difundida en Europa a partir de un influyente artículo de Wilhelm von Waldeyer (1836-1921), quien además acuñó la palabra neurona. Otras técnicas, especialmente las tinciones de Nissl y de mielina iniciaron los estudios de arquitectura cerebral, especialmente cortical, y en humanos, con los Vogt, Brodmann y Paul Fleschig. Este último (1847-1929), anatomista que evolucionó hacia la psiquiatría, propuso varios principios generales de organización anatomofuncional de la corteza cerebral, para los que buscó correlatos clínicos en pacientes neurológicos: las diferencias en curso e intensidad de mielinización revelan diferencias funcionales; las áreas filogenéticamente más nuevas maduran mas tarde, y éstas, que agrupó bajo el término de corteza asociativa, son el asiento de las funciones intelectuales, que emergerían paralelamente a la mielinización de sus tractos conectivos. $\mathrm{Si}$ algo tuvieron en común esos grandes neuroanatomistas de fines de siglo es la pasión por entender cómo es y cómo funciona el cerebro humano. La mayoría, si no todos, fueron además médicos, con ejercicio profesional en alguna parte de su vida. Estos estudios arquitectónicos corticales seguían en buena medida asociados, como lo fueron desde la frenología, al interés por demostrar diferencias cerebrales en distintas razas, en individuos extraordinarios, etc. A partir de la búsqueda de parámetros objetivos de medición del intelecto, y tras la decepción de los abordajes craniométricos, surgiría la primera prueba objetiva de medición intelectual por Alfred Binet (1857-1911) un abogado, que inició pero no terminó la carrera de medicina, lector de la psicología experimental, discípulo de Charcot, y finalmente director de un laboratorio de psicología fisiológica en la Sorbona desde 1894. Con el joven médico Theodore Simon establecieron un test para medir la "edad mental" de los sujetos; pocos años después Wilhelm Stern, psicólogo alemán, la correlacionaría con la edad cronológica para definir el cociente intelectual.

- Aparición y desarrollo de la Neurología en el s. XIX: Probablemente la electroterapia o galvanismo tuvo mucho que ver con la aparición de la Neurología: si los nervios eran excitables eléctricamente y el cerebro producía electricidad que enviaba a los músculos, las enfermedades nerviosas podrían deberse a trastornos en la distribución de la electricidad animal y por tanto serían corregibles con electroterapia. Hasta entonces los tratamientos de afecciones cerebroespinales eran genéricos, y se basaban en la clásica teoría humoral general de la enfermedad. Además, cambió el contexto en que se estudiaba y describían las enfermedades, incluidas las del sistema 
nervioso. La rápida acumulación de conocimientos anatómicos y fisiológicos en un contexto de pensamiento positivista y experimental, de ciencia natural, dio origen a la fisiología moderna con Claude Bernard (1813-1878), y también a la búsqueda de explicaciones fisiopatológicas naturales de las enfermedades neurológicas. Podríamos así situar el comienzo de la nueva epistemología fisiopatológica en el breve estudio de 1817 sobre 6 casos de parálisis agitante del londinense James Parkinson (1755-1824), paleontólogo, médico, cirujano y boticario. Poco después se publicaría el primer Tratado sobre Trastornos Nerviosos, primer texto de Neurología, por John Cooke (1820-1824). Es imposible separar ese desarrollo incipiente de la Neurología, de la propia Psiquiatría y de la neuropatología. Las demencias, las amnesias, las afasias y otros trastornos de funciones relacionadas con el lenguaje, las negligencias, etc. fueron patologías abordadas por clínicos con formación en uno u otro, y con frecuencia varios, de esos campos. Prototipo de esta transgresión de fronteras fue Wilhelm Griesinger (1817-1868), profesor de Psiquiatría y de Neurología en Berlin, neuropatólogo y alumno de Magendie, como Claude Bernard, considerado creador de la Neuropsiquiatría por su actitud integradora de las enfermedades mentales en la medicina neurológica ('Geisteskrankheiten sind Gehirnkrankheiten', 1861), y que fue quien por primera vez asoció las demencias -término introducido por Pinel medio siglo antes- a lesiones orgánicas cerebrales.

- Evolución de la Psiquiatría: Aparecen nuevas nosologías, basadas en cuidadosos estudios de síntomas e historias clínicas, en las bases orgánicas demostrables o supuestas, y en las teorías de la degeneración heredable, con Benedict-Agustin Morel (1809-1873) en Francia, y más tarde Emil Kraepelin (1856-1926) en Rusia y Alemania. A caballo entre el moralismo y el fisicalismo, y con una inspiración indudablemente frenológica, Arthur L. Wigan (?-1847) propondría en las década de los '40 la idea de 2 mentes separadas pero en armonía gracias a la educación, con cierta superioridad de la izquierda (predominio de diestros); cuando la actividad de ambas fuera incongruente se produciría la locura. En paralelo al desarrollo de la focalidad anatomofuncional en Neurología, se multiplicarían en el último tercio del s. XIX las teorías sobre la personalidad, normal y anormal, en relación con la morfología y la asimetría cerebral, invadiendo no sólo la Psiquiatría, sino también la educación, la antropología y la criminología (para Lombroso los criminales natos tendrían hemisferios muy simétricos, más primitivos). La dualidad hemisférica sostendría una dualidad mental. En base a la situación derecha o izquierda de lesiones hemisféricas, y a los cuadros apáticos o emocionales en los correspondientes hemipléjicos, a un supuesto mayor peso del hemisferio derecho en los locos (Luys, 1879), así como a una creciente casuística de histerias, alucinaciones, hipnotismo, etc., creció la convicción de que el hemisferio derecho era melancólico e irracional y el izquierdo moral e intelectual, abriendo especulaciones psicológico-clínicoeducativas sobre si era innata la diferencia o se modificaban los tamaños relativos 
por el aprendizaje y la patología. No es casualidad la publicación de El Extraño Caso del Dr Jekyll y Mr Hyde por R.L. Stevenson en 1886. De ahí nace una larga historia reciente de la personalidad dividida y las disociaciones.

- Psicología moderna: la $2^{\mathrm{a}} \operatorname{mitad}$ del s XIX fue prolífica en la aparición o la consolidación de diversas áreas de conocimiento e investigacion psicológica, a veces en direcciones bien divergentes (psicofísica, psicobiología, psicoanálisis, Gestalt y conductismo), y todas ellas de gran relevancia para comprender las tensiones actuales en que se mueven las ciencias del cerebro y la mente $(3,4,17-21)$.

\section{Psicofísica y Psicobiología}

Gustav Fechner (1791-1887), médico, profesor de Física en Leipzig y más tarde de Filosofía, con el objetivo filosófico de probar la unidad de mente y cuerpo en un panpsiquismo que veía ambos como diferentes aspectos de la misma realidad, estudió en profundidad la sensación y la percepción, iniciando la Psicofísica. Explicó la consciencia en el marco de un paralelismo psicofísico à la Leibniz, pero superando la objección kantiana que negaba la posibilidad de que la Psicología pudiera ser científica. Su continuador, Wilhelm Wundt (1832-1920), que trabajó varios años de ayudante de Von Helmholtz, uno de los más notables fisiólogos del XIX, buscando reducir el psiquismo a la medición de datos fisiológicos exactos, iniciaría poco después la Psicofisiogía. En 1879 fundó en Leipzig el primer laboratorio de psicología fisiológica experimental, un año después de que William James (1842-1910) fundara su laboratorio de psicología experimental en Harvard. Ambos buscarían una conquista de la "totalidad" de la conciencia -proceso, no substancia, para James- y ambos optarían por un pragmatismo operativo, -dualismo fenoménico, no sustancialista-, al no creer factible que nuestro intelecto fuera capaz de ir más allá del establecimiento de correlaciones entre la fisicoquímica del cerebro y el pensamiento y la conciencia, sin poder formular relaciones teóricas explicativas o de causalidad.

Esta psicología fisiológica fue el origen de la Psicobiología, cuya concepción más radical la sitúa en el contexto filosófico que acepta que todas las actividades mentales son (principio de identidad) procesos cerebrales. Nacida así con Müller, Flourens, Broca, Sechenov (quien teorizó que la vida psíquica se basaba en acciones reflejas, incluso para las más complejas asociaciones mentales, sirviendo así de base para la reflexología de Pavlov) y Wundt, en un claro dualismo aún, se eclipsaría en la primera mitad del s XX con el psicoanálisis, la Gestalt y el conductismo, y resurgiría con fuerza en el último tercio del siglo en íntima unión con la Neurociencia.

\section{Gestalt}

La Gestaltpsychologie fue fundada por el austríaco Christian von Ehrenfels (1859-1932), en parte como reacción contra la psicofísica de Wundt y el estructuralismo: el todo es diferente de la suma de las partes, y está por encima de ellas. El movimiento psicológico a que da origen muestra una interesante dialéctica que llega hasta hoy: 
Bunge (17) resume así una visión crítica actual de su historia: "la filosofía subyacente (a la Gestalt) era imprecisa, globalista e intuicionista. Sin duda el globalismo u holismo contiene un importante grano de verdad, a saber que algunas totalidades poseen propiedades emergentes, de las que carecen sus partes. Lamentablemente el globalismo arruina esta tesis verdadera al acompañarla de los dogmas de que el todo siempre precede a sus partes, y de que la emergencia es incomprensible sin ayuda del análisis". Quizá esta es una excesiva simplificación crítica de la evolución de la Gestalt. En la primera mitad del s. XX Kurt Koffka (1886-1941), Wolfgang Köhler (1887-1967) y Max Wertheimer (1880-1943), avanzaron considerablemente las bases teóricas de la perceción o la memoria sobre las relaciones entre la figura y el fondo. El psiquiatra Kurt Goldstein (1878-1965) aplicó la psicología de la forma al análisis de lo concreto y lo abstracto en la esquizofrenia, aceptando la importancia de la patología cerebral subyacente, pero solo como figura (el resto del cerebro proveería del fondo), y consideró que la exclusiva atención médica a ésta suponía un reduccionismo insuficiente para entender al paciente. También sentaría las bases para los modelos de grupo no analíticos, y el desarrollo de la teoría de campo y la dinámica de grupo de Kurt Lewin.

En la Neurología también la Gestalt evolucionó. Constantin von Monakow (1853-1930), profesor de Neurología y neuroanatomista en la Universidad de Zürich, aceptó la localización de funciones y el asiento focal de patologías cerebrales, pero le preocupaba la presencia de efectos funcionales a distancia de los focos lesivos (de ahí su teoría de la diasquisis), y al no poder reconciliar con sus datos el asiento específico de funciones complejas en las áreas asociativas cerebrales, propuso que la memoria y otras funciones mentales superiores requerían de la acción concertada de muy diversas partes del cerebro para expresarse. Esta idea fue desarrollada aún más por Henry Head (1861-1940), quien argumentó que una lesión cerebral que alterara la conducta lo hacía no sólo por eliminación de una función determinada, sino por crear un desorden del sistema en su conjunto. La psicología -¿neurociencia?- experimental de ese tiempo contó con un adalid ferviente de la oposición al localizacionismo rígido en Karl Lashley (1890-1958) quien con Ivory Franz propuso los principios de equipotencialidad (aunque sólo para áreas asociativas y funciones complejas) y la ley de acción de masa, como límite a la equipotencialidad (la pérdida de una función es proporcional a la cantidad de lesión cerebral).

Pero también es cierto que, como en otros marcos heurísticos, ciertas evoluciones teóricas llevaron a su extensión o aplicación a campos mucho más contenciosos: los principios de unidad o totalidad (Ganzheit) de la Gestalt fueron también utilizados a niveles biológico general y cultural, y la interacción de los organismos con el medio se extendió a la interacción de sociedades con sus vecinos, interiores o exteriores, en contextos claramente racistas. Un ejemplo de ello fue la defensa de este principio por Jacob von Uexküll (1864-1944) para la Alemania nazi. 
Hoy los principios gestalticos y la Neurociencia están inextricablemente unidos en el estudio de la percepción, no sólo en el plano psicofisiológico experimental, sino incluso en el abordaje anatómico y neurofisiológico a los mecanismos de la misma.

\section{Psicoanálisis}

El psicoanálisis tiene todos los ingredientes para ser anatematizado por la ciencia biológica moderna. Y de hecho lo ha sido: se han vertido múltiples críticas sobre la validez científica del psicoanálisis desde que tras la II GM se popularizó enormemente no solo en Europa sino también en América. Jacques Monod tildaba a Freud de "anticientífico" y casi "charlatán" (19). Esas críticas se acentuaron desde la aparición de la psicofarmacología a fines de los '50. La desunión no fue unilateral. La reducida lista inicial de patologías susceptibles de abordaje analítico de Freud (21) se expandió considerablemente con los neofreudianos y no-freudianos, muchos disidentes de Freud, para incluir una gran variedad de cuadros psiquiátricos, e incluso diversas enfermedades generales atribuidas a somatizaciones. Este incremento de actividad clínica y terapéutica, junto a densos y a veces divergentes desarrollos teóricos, y el ambiente hostil de otros marcos referenciales hizo, al ver de Kandel $(22,23)$ que los años de auge de la psiquiatría psicoanalítica (1950-80) coincidieran con una actitud general defensiva, de rechazo a las bases biológicas e incluso farmacológicas de la acción sobre lo mental y de exacerbación del desarrollo personalizado de la empatía y el estímulo a la asociación libre del paciente, llegando a la actitud antiintelectual activa de desincentivar la lectura en los residentes de psiquiatría -no sólo la médica o científica, sino incluso la propia psicoanalítica-.

\section{Conductismo}

El conductismo, curiosamente, presenta notables paralelismos con el psicoanálisis, desde una posición teórica radicalmente diferente. Nació con el psicólogo americano John B. Watson (1875-1958) como reacción al mentalismo dominante en primera mitad el s. $\mathrm{XX}$ que se basaba en la introspección como método para determinar el contenido y las operaciones de la consciencia. Propuso que la única psicología científica posible debía confinarse a las respuestas observadas. Más tarde Burrhus Frederic Skinner (1904-1990) llevaría a su apogeo esta línea, y negaría no ya el valor científico de estudiar la consciencia -la mente-, sino la propia existencia de ésta. El fuerte rechazo generado contra el radicalismo reduccionista de Skinner en otros marcos, tanto mentalistas como neurobiológicos, vio su paralelo por parte del conductismo en la negación de la existencia de procesos mentales por el conductismo y su rechazo a considerar necesario o importante buscar explicaciones, teóricas o neurobiológicos, a los fenómenos de conducta que observa.

\section{Dificultades para un reencuentro...}

Como vemos, a lo largo del siglo XX se produjeron, o se consolidaron, muchas separaciones iniciadas en el XIX. Por razones variopintas, y con cursos temporales también diversos.

El éxito de la psiquiatría fundamentada en el psicoanálisis corrió paralelo de un alejamiento de la (neuro)biología. Y esto ocurrió ya con Freud, y por razones bien explicables: a pesar de su familiaridad con la experimentación y la neurología organicista, 
se enfrentó a una dificultad extrema para encontrar explicaciones biológicas al inconsciente, por lo que optó por una opción mentalista: el recurso a la información verbal basada sobre la experiencia subjetiva. Paralelamente, y en un ámbito heurístico radicalmente diferente, el conductismo hizo negación del cerebro y la mente como objetos de interés, reduciendo la conducta a lo observable y medible externamente. Mientras tanto, el localizacionismo cerebral seguía enfrentado a un nuevo holismo y equipotencialismo, tanto en el campo de la neurología y la fisiología cerebral con Franz, Lashley, Von Monakow y Head, como en el ámbito psicológico de la Gestalt. La Neurología, no obstante, ya estaba enmarcada plenamente en el esquema científico del resto de la medicina, adquiriendo una progresiva fundamentación en la bioquímica cerebroespinal y muscular.

En cuanto a la Neurociencia y la Psicobiología, con la licencia de una excesiva simplificación, vemos a partir de la II GM una eclosión de laboratorios de diversas ramas del saber biológico y del estudio de la conducta -especialmente animal-, que se pueblan de miles de científicos con formaciones universitarias diversas (especialmente biólogos, médicos y psicólogos), que se encontraron con -y fueron desarrollando a su vez- infinidad de técnicas y métodos cada día más complejos y poderosos para estudiar un objeto ya abordable en una inimaginada extensión y profundidad: los sistemas nerviosos y las conductas que expresan, desde las moléculas y la biofísica, a las células y los sistemas, y el desarrrollo y el envejecimiento. El éxito de la enorme demanda de formación especializada, y la vastedad intrínseca de los campos de estudio cerebral o conductual tuvieron, paradójicamente, un doble efecto negativo: 1, cada campo específico -neuroanatomía, neurofisiología, neuroquímica, etc.- se consolidaría y expandiría endógenamente, y 2, los nexos con, y el interés hacia las ciencias clínicas declinó tanto que prácticamente habrían desaparecido, de no ser por el creciente desarrollo de la neuro- y psicofarmacología desde los años 50 .

Estas separaciones comparten características más generales en el ámbito de la Medicina, como demuestran varios problemas vigentes hoy que para algunos muestran un preocupante declive actual de la Medicina (24-26): 1, en un número creciente los estudiantes de medicina de últimos años y los médicos jóvenes se sienten tan insatisfechos con su profesión (hasta el $60 \%$ en algunos estudios, en Suiza y el Reino Unido, y probablemente está ocurriendo también en España) que desearían no haberla iniciado, e incluso la abandonan tras años de práctica profesional. 2, un número cada vez mayor de pacientes acude a medicinas alternativas, lo que podría extrañar al producirse en paralelo al desarrollo espectacular de nuevas técnicas y terapias médicas. 3, los costes disparados de la medicina obligan a una racionalización de su aplicación, lo que al mismo tiempo ha burocratizado la relación médico-enfermo a veces de modo muy pernicioso. Una consecuencia nefasta de todo ello es la pérdida del atractivo intelectual y del reto científico que habitualmente aportaba la Medicina. Y 4, se ha producido una progresiva desconexión del médico -en formación y ejerciente- no ya de las ciencias biológicas básicas relacionadas con su especialidad, la Neurociencia o la psicología fisiológica en el 
caso de la Neurología y la Psiquiatría, sino ni siquiera con la investigacion clínica. Entre 1995 y 1998 se produjo un 30\% de caída en el número de licenciados en medicina que obtuvo ayudas de investigacion de los NIH en EEUU. El desinterés de los médicos en la investigación, sea espontáneo o venga impuesto por estructuras poco sensibles a esta necesidad, como en buena medida ocurre en España, tiene un segundo riesgo: desconectar de nuevo lo "objetivo", lo biológico -que puede ser perfectamente estudiable por científicos sin formación ni practica médica- de lo relacional, subjetivo e individual.

\section{Pero reencuentro, al fin...}

Una breve ojeada a los textos actuales de Psiquiatría o de Neurología demuestra el hecho quizá ya irreversible de la incorporación de la Neurociencia moderna al armamentario intelectual de la clínica. En la $7^{\mathrm{a}}$ edición del Comprehensive Textbook of Psychiatry, de Kaplan \& Sadock (27), casi el 17\% de sus 3344 páginas está dedicado a la neurobiología, neurofisiología/neurología y farmacología no específicamente psiquiátrica. Esta integración en los textos ha sido precedida de diversos desarrollos, todos ellos aún vivos y cambiantes. Podríamos resumirlos en 3 apartados: la adquisición de un nuevo marco intelectual para la comprensión del cerebro, sano y enfermo, la aparición de una Neurociencia -en singularmoderna en el último tercio del s. XX, y la progresiva interpenetración de lo clínico y lo básico en el campo del cerebro y la mente $(17,19,22,23,28-30)$.

- El desarrollo de un nuevo marco intelectual para la comprensión del cerebro, sano y enfermo es un reto que deberán asumir quienes quieran liderar intelectualmente ese campo, y quienes hayan de educar a otros en el mismo. Y sólo podrá hacerse desde una expansión de las perspectivas intelectuales propias de cada campo. Estas serían algunas ideas para tal expansión:

- Hay que recuperar el contacto con y la perspectiva desde la filosofía, aunque solo sea para poder entender qué es hoy la ciencia, y aceptar críticamente que la ciencia no está libre de controversias, como atestiguan los debates sobre los modelos del átomo, la materia y el universo, el problema mente-cerebro, la naturaleza científica o metafísica del neodarwinismo, la relación entre ciencia y valores/ética, o la propia dificultad de aceptar esta propuesta. Desde el positivismo de Comte hasta el interaccionismo antipositivista de Popper, el ámbito en que se desenvuelve la ciencia ha cambiado apreciablemente, aunque ninguno de los dos es proclive a ese contacto. Para el primero la ciencia marcaría la cúspide del pensamiento humano, en todos los aspectos, incluida la ética, y su instrumento central sería la propuesta y verificación de leyes naturales a partir de datos, pero la filosofía como tal desaparecería, conviertiéndose sólo en la reflexión sobre la ciencia. Popper, en cambio, con sus principios de demarcación y de falsabilidad apriorística de las hipótesis puso importantes límites al ámbito de la ciencia; descalifica como no científicos nuevos marcos ideológicos en la sociología (Marx) y la psicología (Freud), y excluye del ámbito científico las humanidades, incluyendo la propia filosofía $(3,17,31,32)$. 
- Es preciso advertir que, en cualquier ámbito del conocimiento, los datos nunca son absolutamente "objetivos", a menos que reduzcamos los criterios de objetividad de los hechos naturales como Skinner reducía los criterios de definición de la conducta. A pesar de la extendida predilección de los científicos por quedarse en los hechos observados, incluso exigiendo no pasar a la especulación teórica, Popper, el filósofo más influyente en el pensamiento científico actual sin duda, advierte que la observación debe suceder a la experiencia y la hipótesis, y que todos los conceptos están cargados de encuadre teórico: no hay nada de "hechos, y solo hechos".

- Hay que conocer y reflexionar sobre las analogías, paradigmas o metáforas en que enmarcamos nuestras observaciones, experimentacion y conocimiento del cerebro y la mente hoy, y para ello es conveniente conocer cuáles han sido, y por qué, los utilizados en tiempos pasados [cf. refs. $(4,16,17,20,33)]$. No es en absoluto irrelevante para nuestro modo de pensar actual la evolución que éstos han sufrido, desde la teoría pneumatica presocrática a la metáfora eléctrica, cuando tras el descubrimiento de la electricidad animal se sustituyeron los humores por electricidad (inspiración del monstruo de Frankestein, animado por descargas eléctricas que dan vida); la metáfora telefónica y su incipiente conexionismo; la muy popular hoy metáfora computacional, a partir de Gödel, Turing y von Neumann antes de la II GM, que ha evolucionado al actual modelo de redes neurales, con los procesamientos distribuidos, códigos de informacion diversos, y analogías interpretativas del hardware y el software, etc. Y la red de redes, especie de supraorganismo que vincula las computaciones individuales.

- Hay que evitar reduccionismos sustancialistas u ontológicos. El problema del reduccionismo, al menos en Biología, sigue planteando muchas cuestiones de gran interés teórico y práctico $(5,17,34,35)$, entre los que no es la menor la del poder explicativo de cada nivel posible de reducción (ontológico, gnoseológico, metodológico, etc.) para cada nivel de complejidad de los fenómenos (desde la física subatómica a la sociología). Tras las actitudes reduccionistas más claras aparece con frecuencia el intento de alcanzar teorías unitarias. Intento realmente atractivo, pero con una historia cargada de promesas incumplidas, especialmente cuando en la unificación se quieren agrupar niveles de organización o complejidad sustancialmente distintos. Tal deseo se percibe con frecuencia en textos de filósofos de lo mental $(36,37)$, pero ni es algo logrado ni necesariamente es algo perseguible a expensas de imponer reducciones cuya capacidad explicativa es, al menos, insuficiente. Ante la existencia de interrogantes de calado es mejor dejar puertas abiertas que cerrarlas en falso o prematuramente.

- El llamado problema mente-cerebro es un campo filosófico de especial interés para la Psicología y la Neurociencia, y en el que se aprecian posiciones de toda índole. Tras la muerte de Sir John Eccles (1903-1997), extraordinario neurofisiólogo y 
último adalid del dualismo sustancial, el jardín de los debates sobre el tema se encuentra hoy en plena floración, desde libros a simposios en Internet, con elaboradas pugnas desde las posiciones de identidad psiconeural reduccionista acérrima (los estados mentales son estados cerebrales (Dennett, Crick, Churchland) hasta diversas interpretaciones sobre una interrelación bidireccional entre ambos estados (Thomas Nagel, Searle). Como trasfondo persiste hasta hoy la sospecha de que podríamos no llegar nunca a comprender del todo la mente: sería un misterio intrínseco, no provisional (20). Esta sospecha se articuló ya claramente en el dualismo fenoménico de Tindall o de W. James, quien temía que careciéramos del órgano intelectual necesario para razonar sobre una posible correlación entre un pensamiento y una acción molecular definidas, aunque pudiéramos demostrar que se dieran simultáneamente. Extendida una duda similar a todo el conocimiento de la naturaleza por Max Planck ("Science cannot solve the ultimate mystery of Nature. And it is because in the last analysis we ourselves are part of the mystery we are trying to solve"), persiste hoy en diversos científicos y filósofos esa incertidumbre radical sobre nuestra capacidad cognitiva. Tal actitud ha sido criticada desde campos neurobiológicos y filosóficos como negadora de una realidad que es material, y sólo material, o, en afortunada expresión de Angel Rivière, como un paralizante pesimismo epistemológico (20). Pero también es probable que otras posiciones modernas hayan sido encuadradas negativamente en el mismo esquema, cuando en realidad han dejado puertas abiertas a análisis probablemente más explicativos y creativos. Así, Thomas Nagel propone la necesidad de un tipo de teoría radicalmente nuevo y diferente de las usadas en la física para explicar la relación entre la conducta, la conciencia y el cerebro: nuestro conocimiento actual sobre el problema mente-cuerpo sería de naturaleza empírica y correlacional, no causal y teórica; los estados mentales, los fenómenos conductuales y las actividades neurofisiológicas están relacionados entre sí, pero no son equivalentes, ni analizables desde un punto de vista de la física de éstas últimas; sugiere que la objetividad de nuestas descripciones del fenómeno se conseguiría a expensas de sacrificar, por imposibilidad de ser reconocida científicamente, la subjetividad $(20,38)$. La subjetividad se caracteriza por conciencia personal de un yo individual, intencionalidad, elaboración personal de significados y un cierto grado de libre albedrío. Aunque solo fuera todo esto, ciertamente parece difícil de abordar por el estudio de ondas cerebrales, vectores poblacionales y modelos computacionales. Pero no por ello ha de quedar irremisiblemente fuera del abordaje científico. Eso sí, es probable que precise paradigmas nuevos. Así, para Gerald Edelman (19) "la incorporación -embodiment- del yo impone límites inevitables. El deseo de transgredir esos límites crea contradicciones..., porque, pasado un cierto punto, la mente se sitúa más allá del alcance de la ciencia, al menos en sus creaciones como mente individual... La razón de esos límites es clara: las formas de incorporación que conducen a la consciencia son únicas para cada individuo, únicas para su cuerpo y su historia individual". Para Edelman la comprensión del cerebro -especialmente 
el humano- enfrenta a la ciencia que conocemos hoy al reto de la individualidad de la mente, con su vertiente emocional, su intencionalidad y su adscripción de significados, incluidos los valores. Es un reto que difícilmente puede ganar hoy todavía. Y sin embargo, desde su "realismo cualificado" y "materialismo sofisticado" su propuesta es opuesta a todo pesimismo: para él la investigación en Neurociencia buscando nuevos paradigmas podrá llega a modelar cada vez más aspectos de las funciones mentales complejas que permitirán plantear y probar hipótesis en las que pueda incluirse ese aspecto de la individualidad histórica de cada cerebro.

- Por último, es hoy posible, y necesario, "devolver la mente a la naturaleza" en expresión de Whitehead (19). Es posible desarrollar una epistemología que, con el objetivo de llegar a explicar cómo conocemos y cómo tenemos conciencia, se fundamente en la evolución, la biología del desarrollo y las relaciones estructura/función de nuestro cerebro. Y hemos de extender ese marco epistemológico a las "enfermedades mentales". No se justifica ya mantener un cercado de enfermedades puramente mentales o psicológicas, reeditando la separación de un dominio funcional contrapuesto a un dominio orgánico. Eric R. Kandel reclama específicamente en dos recientes y debatidos artículos en el American Journal of Psychiatry $(22,23)$ un diálogo genuino entre Biología y Psicoanálisis como requisito para poder llegar a alcanzar una comprensión coherente de la mente. Aunque hay que ser consciente de la dificultad que ello entraña, no sólo por la genérica inercia conceptual (36), que se opone a la incorporación de nuevos prototipos heterodoxos respecto a los prototipos antiguos, más encardinados en el conocimiento de sentido común, sino porque realmente ambos marcos heurísticos han seguido por mucho tiempo sendas muy divergentes.

- El origen más comúnmente admitido de la Neurociencia moderna, fue el establecimiendo del Neurosciences Research Program en el MIT por Frank O. Schmitt en 1962, al que atrajo a científicos de muy diversas áreas, con el explícito objetivo central (Schmitt, 1963, informe sobre el programa, cit. en ref. (28)) de explotar al máximo los abordajes clásicos de fisiología y conducta y combinarlos con la potencia técnica y conceptual de la física, la química y la biología molecular, con la perspectiva de conseguir "avances revolucionarios enlacomprensión de lamentehumana”. ElNRP patrocinó simposios y reuniones de cuyas deliberaciones nacieron 4 voluminosos textos entre 1967 y 1979, que recibieron como título genérico "The Neurosciences: First, Second, etc... Study Program". El NRP de Schmitt, tuvo un claro precedente casi 10 años antes, con un especial interés aquí: David McKenzie Rioch, psiquiatra y neuroanatomista, congregó en su División de Neuropsiquiatría del Walter Reed Army Institute of Research de Washington DC a varios psiquiatras, psicólogos experimentales, neuroendocrinólogos, anatomistas y neurofisiólogos con el objetivo de abordar de modo interdisciplinar el estudio del cerebro y la conducta. Uno de los primeros estudiantes postdoctorales en ese grupo fue David Hubel, que sería galardonado con el Premio Nobel de Fisiología y Medicina en 1981, en lo que fue la primera "hornada" de estos premios a neurocientíficos en la era de la Neurociencia. 
Dos pasos ulteriores fundamentales en la aparición de la Neurociencia moderna fueron la creación del primer Departamento multidisciplinar de Neurociencia (realmente Neurobiología) por Stephen Kuffler, en la Universidad de Harvard en 1967, como evolución de un Departamento previo de neurofisiología. Y la creación en 1969 de la Society for Neuroscience $(S F N)$ por iniciativa de un reducido grupo de investigadores, principalmente neurofisiólogos de sistemas, psicólogos experimentales y psiquiatras, bajo el liderazgo de Ralph Gerard, un bioquímico de la Universidad de Irvine, introductor del término Neurociencia, en singular (30).

De la evolución posterior de este proyecto son buenos testimonios la proliferación de centros, departamentos e institutos de Neurociencia o Neurobiología en EEUU y otros países, el espectacular y mantenido crecimiento en la afiliación a sociedades de Neurociencia, especialmente la SFN (que se acerca hoy a los 30.000 miembros), y las sociedades nacionales europeas federadas en la FENS, y la multiplicación de revistas científicas generales o especializadas de Neurociencia: antes de 1970 no se podían encontrar más de 6 revistas no clínicas de investigación del sistema nervioso. Hoy el Institute for Scientific Information de Filadelfia recoge 200 bajo el epígrafe de Neurociencias. ¿Por qué es tan fundamental y qué tiene de especial la integración y la multidisciplinariedad en el estudio del sistema nervioso, cuando también se demanda, y se produce, en otras áreas de la biología? En primer lugar, es un paso imprescindible para devolver no solo la mente, sino el cerebro, a la biología. En cualquier órgano existen procesos de proliferación y muerte celular, mecanismos de señalización intra- e intercelular, relaciones endocrinas y paracrinas con el medio, etc. Pero, quizá con la excepción parcial del sistema inmune, el sistema nervioso se especifica por sus propiedades de aprendizaje, memoria y plasticidad, sus peculiares y complejísimos procesos de comunicación celular, a nivel individual y poblacional, y, sobre todo, por la conducta que todo él sustenta. Y en segundo lugar, más que en ningún otro órgano o sistema, el reduccionismo metodológico es estéril al aplicarlo al cerebro, la conducta o la mente; no ya porque un solo abordaje conceptual no logrará resolver los problemas que plantea, sino porque es fácil caer en la tentación de convertir en problema científico lo que solo es una ventana de observación con la que uno se encuentra cómodo. La necesaria limitación intrínseca a la aplicación de un método y al desarrollo de una experiencia científica individual no exime de la obligación de definir problemas reales en neurociencia, y de no crear pseudoproblemas. El proceso de constitución de la Neurociencia muestra algunos jalones de particular relevancia:

- Las ciencias básicas "clásicas" que estudiaban el sistema nervioso y la conducta fueron las primeras en congregarse en torno al nuevo marco intelectual integrador de la Neurociencia. A partir de ellas se desarrollaron los capítulos de Neurociencia celular y de sistemas, los primeros pasos de la Neurociencia del desarrollo ontogénico, y la Neurociencia de la conducta, ésta última fundamentalmente desarrollada por psicólogos de corte conductista o neurofisiólogos que, ya a fines de los años 60, registraban 
la actividad neuronal en primates u otras especies despiertos y durante la ejecución de tareas sensoriales o motoras, combinando así la psicofisiología con la electrofisiología. Pero pronto la Neurociencia atrajo rápidamente a la práctica totalidad de áreas de la ciencia natural y la psicología -y de otros campos de la tecnologíía que tenían algo que preguntarse -aunque en varios casos poco que contestar aún - sobre el cerebro y la conducta. Así emergieron nuevos capítulos en la Neurociencia: el primero, a fines de los años 70, fue la Neurociencia molecular, a partir de la biología y la genética molecular. Supuso -y supone hoy- una profundización fecundísima en los mecanismos subcelulares que sustentan el desarrollo y la herencia celular, la señalización intracelular y la comunicación intercelular. No por ello es hoy la NB molecular simplemente un capítulo especial de la biología molecular en general. Hasta el nivel más reducido de las moléculas y los genes está cargado de epistemología cuando se trata del cerebro y la mente, como prueban los nuevos materialismos neurobiológicos de Francis Crick, Rodolfo Llinás u otros en estos asuntos.

- Un segundo salto de gran trascendencia para la Neurociencia fue la aparición de la Neurociencia cognitiva, a partir de una oportuna combinación de una serie de avances metodológicos neurocientíficos y ciertas conquistas -o redescubrimientosconceptuales, sobre todo en la psicología de los años 70 (18, 39-42). No es de extrañar que primero tuviera que re-surgir una Psicología cognitiva (con Ulric Neisser en Cornell, Noam Chomsky en MIT o George Miller en Harvard). En ella se da un valor central para entender la construcción de la percepción, el aprendizaje y la memoria no sólo al conocimiento de las propiedades físicas de los estímulos o la fisiología de la transmisión neural, sino también a la actividad mental del que percibe, aprende y memoriza. Desde la Psicología esto suponía estudiar el flujo y el procesamiento de información, desde la transducción del estímulo en los receptores hasta sus efectos en el cerebro que conducen a la memoria, el pensamiento o la conducta expresada emocional o motrizmente. Esa información circulante, interactuando con la propia actividad cerebral, generaría representaciones de la información en las diversas regiones cerebrales en forma de actividad neural de algún tipo. Más tarde se vería que en muchos casos existen también correlatos estructurales de las mismas. No es de extrañar que la Neurociencia cognitiva tardara aún 2 décadas en aparecer. A principio de los años 70 el único análisis biológico posible de esas representaciones era el aplicado en los pioneros estudios de Mountcastle en el sistema somestésico, Hubel y Wiesel en el visual o Evarts en el sistema motor, todos basados en los patrones de actividad neuronal unitaria o multiunitaria, y casi exclusivamente midiendo la intensidad y las correlaciones temporales de la frecuencia de disparo neuronal con las tareas realizadas. Además, el desarollo teórico del procesamiento de esa información contaba con un aún muy limitado repertorio de modelos computacionales, casi exclusivamente lineales, que fueron útiles para entender sólo algunos modos limitados de computación neuronal. Realmente el progreso posterior de los modelos computaciones en el campo de las 
redes neurales y en otros procedimientos no lineales debe mucho a la interacción entre fisicos/informáticos, y psicólogos y neurocientíficos, que aportaban las bases biológicas y el diseño de tareas y análisis de conducta. Pero los métodos que realmente dieron el empuje definitivo a la Neurociencia cognitiva fueron los de neuroimagen: la tomografía axial (TAC), la resonancia magnética (RM), y especialmente la RM funcional (FMRI) y la tomografía de emisión de positrones (PET), y, más recientemente, la magnetoencefalografía (MEG), permitieron abrir ventanas de observación del cerebro funcionando en humanos despiertos y activos, de modo nada o mínimamente invasivo. Entre sus principales aportaciones conceptuales puede destacarse que permiten realizar comparaciones de datos de actividad cerebral en humanos y en animales experimentales, especialmente primates, en estudios de percepción; permiten ver datos espaciales (activaciones regionales) y temporales en relación con funciones mentales complejas; muestran cambios transitorios o permanentes en situaciones "plásticas" de aprendizaje, y de hiper- o hipoestimulacion; y son de extraordinaria utilidad en el diagnóstico y seguimiento de patologías diversas neurológicas y psiquiátricas.

- Un tercer salto, de grandísimo calado, fue la incorporación plena de la genética molecular a la Neurociencia en los años 80 . Hasta entonces se habían realizado ya incursiones pioneras de gran importancia en el uso de abordajes genéticos para el estudio del cerebro y la conducta desde los años 60. En esa década Seymour Benzer buscó mutaciones en Drosophila que afectaran la conducta: sus estudios serían semilla de la notable expansión del uso de este modelo animal para estudios de canales iónicos, cadenas de señalización intracelular, desarrollo neural y genética de la conducta. Simultáneamente Sydney Brenner introduce el nematodo C. elegans como modelo de estudio de desarrollo y neurobiológico; y con Hendrik Van der Loos, Thomas Woolsey y Pasko Rakic comienza el uso de mutantes espontáneos de ratón para estudios de conducta y desarrollo neural.

- Neurociencia, Neurología y Psiquiatría: La apertura de la Neurociencia a la clínica no es nueva. Desde su inicio incorporó apartados dedicados a Neurociencia clínica, durante mucho tiempo muy minoritarios, pues los puentes históricos entre ambos bandos eran tenues e inseguros, y se limitaban a algunos aspectos más o menos aplicativos de la psico- y neurofarmacología. Pero durante la década de los 90, -la Década del Cerebro- se produjo una explosión de interés: En la reunión anual de la SfN en 1990, menos del 1\% de comunicaciones presentadas se referían a la patología neurológica o mental y el envejecimiento cerebral; en la de 1999, esa cifra superó el 20\%. Las razones principales de este auge podrían encontrarse en: 1, desarrollo de fármacos cada vez más específicos que actúan sobre mecanismos complejos en procesos de daños isquémicos cerebrales, dolor, trastornos afectivos y psicosis, etc; 2, desarrollo de las técnicas de neuroimagen; 3, desarrollo incipiente de prótesis 
sensoriomotoras efectivas; 4, aplicación de injertos biológicos para reparación de defectos o patologías, y 5, muy principalmente, los descubrimientos que la genética molecular aportó en el campo de la patología neurológica con base genética. Es difícil decidir donde se encuentra un mayor entusiasmo sobre la invasión de la genómica en la Neurociencia, si en los neurocientíficos o en los neurólogos y -dentro de poco- los psiquiatras. La posibilidad de identificar, clonar y secuenciar genes relevantes para la patología neurológica y psiquiátrica introduce no solo nuevas herramientas de diagnóstico y, en un futuro razonable, tratamiento (incluido el desarrollo de tratamientos genéticos y de terapias individualizadas de acuerdo con los genomas propios), sino que es muy previsible que ello conduzca a una redefinición nosológica de la patología neurológica, en la que los síntomas y la neuropatología cedan el puesto de criterios dominantes de clasificación a los mecanismos genéticos, moleculares y celulares de cada enfermedad, como de hecho ya ha comenzado a ocurrir en algunos grupos de patologías (canalopatías y enfermedades mitocondriales, cromosoma X lábil, enfermedades lisosómicas, etc) $(28,43,44)$. Un punto de partida se sitúa en la primera clonación exitosa de un gen asociado a una patología neuromuscular, la distrofia muscular de Duchenne, por el grupo de Louis Kunkel. Un segundo avance en la neurogenética de enfermedades monogénicas de gran repercusión fue el aislamiento del gen responsable de la enfermedad de Huntington. Este avance tuvo un importante prolegómeno: el extraordinario estudio familiar realizado por el grupo de Nancy Wexler sobre los miembros de una familia extendida cerca del lago Maracaibo, con el linaje trazado hasta una mujer de principios del S. XIX (de 3000 pacientes vivos de este grupo hoy 100 sufren la enfermedad). En muestras de DNA de estas personas Wexler y James Gusella localizaron en 1983 el gen responsable en el cromosoma 4. Diez años después aislaron el gen y mostraron que codificaba una proteína de $350.000 \mathrm{kDa}$, la huntingtina, y que presentaba una característica peculiar: un número muy superior al normal de tripletes de nucleóticos repetidos (CAG, que codifica glutamina), y que había una relación entre el número de tripletes y la edad de aparición de la enfermedad. Esto ya se había observado poco antes en el síndrome del cromosoma $\mathrm{X}$ frágil, y posteriormente se vería en otras patologías neurológicas genéticas. Un ejemplo de la rápida y eficaz colaboración entre la clínica y la neurogenética molecular lo resume Harold Varmus, ex-director de los NIH, cuando relata cómo en 1996 un grupo de neurólogos que estudiaban una familia italiana con enfermedad de Parkinson familiar se encontraron con otro grupo de genetistas en una reunión en los NIH. Nueve días después se encontró un gen asociado a esa forma familiar del Parkinson en el cromosoma 4, que se aisló varios meses después, descubriéndose en él una mutación específica. La proteína fabricada por ese gen se identificó rápidamente, se produjeron anticuerpos contra ella y se localizó en los cuerpos de Loewy, característica neuropatológica conocida desde antiguo y asociada a las formas familiar y no familiar de enfermedad de Parkinson. En cuanto a la Psiquiatría, partía con mayor desventaja para este acercamiento. Su 
nosología carece de algo bien asentado clásicamente en la Neurología: la localización de las lesiones o las patologías degenerativas en regiones más o menos específicas del sistema nervioso. Recientemente esta necesidad se ha explicitado con afirmaciones tajantes: "By contrast (with neurological disorders) we know frustratingly little about the anatomical substrata of most psychiatric diseases. A reliable neuropathology of mental disorders is...severely needed" (43). La evidencia de factores genéticos en varias patologías psiquiátricas mayores (esquizofrenia, psicosis $\mathrm{M}-\mathrm{D}$, trastornos obsesivo-compulsivos, etc.) es incuestionable, pero por hoy la búsqueda de polimorfismos genéticos asociados ha dado pocos resultados relevantes.

\section{4.... En un terreno abrupto.}

La exposición de buenos deseos de integración puede ser atractiva, y hasta políticamente correcta, pero acaba siendo estéril si no se enmarca adecuadamente en el tumultuoso contexto de la realidad. La progresiva integración de la Neurología y la Psiquiatría con la Neurociencia bien puede verse obstaculizada, cuando no desprestigiada, por algunos escollos:

- El marco intelectual, o proceso heurístico en el que se mueve cada disciplina -o subdisciplinas- tiene componentes fácilmente objetivables y en gran medida compartibles, pero otros generan disensiones, a veces profundas, y generalmente por falta de reflexión crítica sobre la génesis, evolución y situación actual del propio marco. Existen arraigados clichés que marcan territorios desde dentro y desde fuera y se alimentan normalmente del desconocimiento, no ya del otro, sino de las posiciones de fondo en las que uno mismo se encuentra.

- Hoy plantea más riesgos de incomunicación el reduccionismo en la ciencia del cerebro (la mente no es más que el cerebro, éste no es más que redes, o genes, etc), y también en la clínica (ya es frecuente olvidar que no hay enfermedades, sino enfermos), que las actitudes moderadamente escépticas sobre la amplitud de la capacidad explicativa de los abordajes intelectuales con que contamos hoy. El carácter individualizado y personal del cerebro y la mente inclina más a la posición unamuniana que enfrentaba al cartesiano cogito ergo sum, un sum, ergo cogito: solo si Descartes, la persona, existe, puede pensar. Y eso pone en aprietos los intentos reduccionistas que quieran salirse del plano metodológico o gnoseológico para dar explicaciones ontológicas de validez universal con poca consideración a los diferentes niveles de organización y complejidad.

- Existe un amplio registro histórico de abusos derivados de la extensión, prepotente y acrítica, de conceptos o actitudes muy "propias" de cada área: la poca atención al tratamiento del paciente en la clínica decimonónica de Osler, el racismo sobre bases biológicas, el movimiento eugenésico, la eliminación del campo de valores de la ciencia y la técnica, etc. Es fácil tomar la parte por el todo para marcar mejor un territorio defensivo. 
- El volumen de conocimientos, la complejidad de las técnicas y los métodos, y un innato miedo a meterse en terrenos inciertos, avalan la tendencia a quedarse en marcos propios, familiares, satisfactoriamente explicativos. Además es difícil el ejercicio de la autocrítica y la heterocrítica en la valoración de abordajes y campos, pero ambas son imprescindibles para un progreso hermenéutico. Dos ejemplos podrían ser el largo enfrentameiento entre el localizacionismo y el holismo/gestalt, hoy felizmente superado en un marco de integración neurocientífica de enorme interés para la comprensión de mecanismos básicos de las funciones cerebrales; y el diálogo entre las psicofarmacología y la psicoterapia, ya muy evolucionado, pero sobre el que no creo equivocarme si afirmo que aún hoy existen posiciones muy recalcitrantes de incomprensión mutua [véase, p.ej., ref. (45)].

- Existen obstáculos prácticos para realizar la aproximación entre los campos. Probablemente mucho menores en los países anglosajones, y especialmente EEUU, mucho más dinámicos en la promoción de ideas nuevas y en la creación y consolidación de fusiones intelectuales bizarras, en nuestro país constituyen sin embargo muy serias barreras la inercia histórica ante la ciencia, la falta de estímulo a la creación científica en las formaciones profesionales relacionadas con la medicina, la dificultad de crear grupos amplios bien integrados y financiados, etc.

- La ciencia en general, no sólo la Neurociencia, experimenta en este inicio del s. XXI una popularidad y atención inéditas. Su extensión, sus logros y la globalidad en las comunicaciones la han hecho llegar hasta los últimos rincones del mundo y la han convertido en frecuente referente o contraste de conocimiento, fiabilidad y prestigio. No debe ignorar la ciencia el riesgo de morir de éxito. Aunque es un tema que requeriría un estudio aparte, baste enunciar aquí algunos soportes reales de ese riesgo: en buena medida la profesionalización de la actividad científica convierte a ésta en un trabajo más, marginando su raíz reflexiva, estudiosa y creativa; la competitividad científica cada vez se parece más a la industrial y menos a la académica; el mensaje que los sistemas de evaluación de la producción (nefasto término) científica envían a los jóvenes investigadores es claro: sólo sobreviviréis si aumentáis vuestro factor de impacto lo antes posible; las líneas de investigación priorizables (necesaria y peligrosa estrategia) serán las más vendibles, especialmente a la industria y a los administradores de los recursos científicos, en base a expectativas de producción a corto plazo de remedios, patentes y artefactos; esta evolución en las actitudes ante la ciencia la estamos ya percibiendo en la Universidad; y se está abusando de las promesas ante el público por parte de científicos o poco honestos, o poco realistas, o demasiado atosigados por la presión competitiva, pero que en cualquier caso hacen un flaco favor al progreso científico. 


\section{Una coda española}

En España el número de grupos que investigan en Neurociencia en los Hospitales, el CSIC y la Universidad, así como el de proyectos financiados, se ha expandido considerablemente en los últimos años, recabando una fracción importante de las inversiones en investigación biomédica, y en diversas áreas los neurocientíficos españoles poseen un envidiable reconocimiento internacional. Pero España adolece de algunas carencias generales crónicas que siguen comprometiendo su capacidad de alcanzar un grado de actividad y de competitividad más acorde con su nivel educativo y económico. En primer lugar, la inversión pública en investigación en general está aún muy por debajo de la media europea. Además, aunque ha mejorado el tratamiento fiscal de las fundaciones y donaciones, aún queda un largo camino por recorrer en la mentalización del público y de nuestros administradores para que las inversiones en investigación se promuevan como un auténtico bien social que debe superar su casi testimonial nivel actual. Es preciso asimismo renovar profundamente los mecanismos actuales de formación y contratación de investigadores en la Universidad, el CSIC y el Sistema Nacional de Salud. La falta de condiciones y de estímulo a la investigación biomédica en el sistema hospitalario está ya produciendo generaciones de médicos para los que la formación y la dedicación compartida a una actividad investigadora no pasa de ser un lujo o una anécdota. Y la creación y renovación positiva de grupos de investigación en el Consejo o la Universidad sólo pueden pasar por la introducción de mecanismos nuevos, ágiles y pragmáticos de contratación y promoción de investigadores y profesores, y por una renovación a fondo de las infraestructuras de investigación y el personal técnico especializado.

La Década del Cerebro, aunque no declarada oficialmente en nuestro país, sirvió de amparo a un notable crecimiento de la investigación neurocientífica. La Sociedad Española de NeuroCiencia (SENC) se consolidó en esa década y apostó decididamente por establecer vínculos con otras sociedades más próximas a la clínica. Además los neurocientíficos españoles están aprendiendo la importancia de alejarse periódicamente de su laboratorio para meditar sobre lo que hacen y sus mensajes. Hoy está claro que la consecución de mayores recursos para las investigaciones cerebrales no va a depender sólo de su fundamentación en argumentos sólidos, razones objetivas y definición de intereses a largo plazo. Todo ello ha de ser, además, expuesto ante un público cada vez más amplio, con capacidad y voluntad de estimular a los organismos que pueden patrocinar y financiar estos recursos, que ya no son solo las administraciones nacional y autonómicas sino, de modo aún tímido pero creciente, las iniciativas privadas y las asociaciones de pacientes, enfermos o familiares de enfermos afectados por graves patologías neurológicas y psiquiátricas. $\mathrm{Y}$ en esta dirección nos hemos movido recientemente los neurocientíficos españoles.

En 1998 un amplio grupo de neurocientíficos presidido por Alberto Ferrús, José López Barneo, Manuel Nieto-Sampedro y Luis Puelles mantuvo un debate con representantes de los medios de comunicación en Madrid, dentro del marco del Día Europeo del Cerebro instituido por la Alianza Dana Europea. En esa reunión se presentó 
un manifiesto en el que se insistía en la íntima ligazón de la investigación en Neurociencia con la docencia universitaria y la asistencia clínica, y en la necesidad de dar respuesta desde la Neurociencia a las demandas de los grupos de pacientes y familiares afectados por las diversas enfermedades neurológicas, facilitando además la transferencia eficaz de conocimientos desde los laboratorios de investigación a los centros de aplicación.

Más recientemente aún, por iniciativa de la SENC un amplio grupo de sociedades científicas relacionadas con el mundo de lo neurobiológico suscribió la creación del Foro Neuro XXI, con la voluntad de aunar y hacer más eficaces los esfuerzos por desarrollar las investigaciones cerebrales en el Sistema Nacional de Salud. Terminaré esta exposición con unas consideraciones que sirvieron de soporte a la redacción del manifiesto de este Foro:

“...Revelaría un escaso alcance de miras por parte de los investigadores o de las instituciones ... que los financian condicionar el progreso de la Neurociencia a la "producción" de remedios y patentes. En primer lugar, porque detrás de las innovaciones terapéuticas y tecnológicas existe un extraordinario -y con frecuencia ignorado- esfuerzo de investigación fundamental. Y en segundo lugar porque, como en otras áreas de la Ciencia, existen réditos a más largo plazo, menos pragmáticos, más conceptuales, pero cuya simple expectativa constituye un pilar fundamental para el mantenimiento de una atmósfera adecuada que propicie el gusto por la actividad creadora, y la educación e incorporación de jóvenes a esta ilusionante tarea. Sin criterios exclusivistas ni reduccionistas, el avance del conocimiento sobre nuestro cerebro modificará -de hecho lo está haciendo ya- nuestra comprensión global del ser humano, como individuo y como sociedad, ayudará a orientar nuevas pautas en la educación, y probablemente contribuirá a mejorar las prioridades que guían la conducta humana". 


\section{BIBLIOGRAFÍA}

(1) Martín Santos, L., Apólogos, Barcelona, Seix Barral S.A., 1970.

(2) Laín Entralgo, P. y otros, Historia Universal de la Medicina, Barcelona, Salvat, 1973.

(3) Marías, J. y Laín Entralgo, P., Historia de la Filosofía y de la Ciencia, Madrid, Ed. Guadarrama S.L., 1964.

(4) Finger, S., Origins of Neuroscience. A History of Explorations into Brain Function, New York, Oxford Univ. Press, 1994.

(5) Wartofsky, M. W., Introducción a la filosofia de la ciencia, Madrid, Alianza Universidad, 1973.

(6) Lazinier, E., Auguste Comte (1798-1857) et le Positivisme, http://membres.lycos.fr/clotilde/ home.htm, 2000.

(7) Colp, R., Jr., "History of Psychiatry”. En: Sadock, B. J. y Sadock, V. A. (Eds.), Kaplan \& Sadock's Comprehensive Textbook of Psychiatry, Philadelphia, Lippincott Williams \& Wilkins, 2000.

(8) Zola-Morgan, S. "Localization of brain function: The legacy of Franz Joseph Gall (1758-1828)", Annual Review of Neuroscience, 1995, 18, 359-383.

(9) Shorter, E., A History of Psychiatry: From the Era of Asylum to the Age of prozac, New York, John Wiley \& Sons, Inc., 1998.

(10) Caine, E. D. y Lyness, J. M., "Delirium, dementia, and amnestic and other cognitive disorders". En: Sadock, B. J. y Sadock, V. A. (Eds.), Kaplan \& Sadock's Comprehensive Textbook of Psychiatry, Philadelphia, Lippincott Williams \& Wilkins, 2000.

(11) Dobzhansky, T., Ayala, F. J., Stebbins, G. L., y Valentine, J. W., Evolution, San Francisco, Freeman \& Co., 1977.

(12) Montalenti, G., "Desde Aristóteles hasta Demócrito vía Darwin: Breve perspectiva de un largo recorrido histórico y lógico". En: Ayala, F. J. y Dobzhansky, T. (Eds.), Estudios sobre la filosofia de la biología, Barcelona, Ariel S.A. 1983.

(13) Stebbins, G. L. y Ayala, F. J. "The evolution of darwinism", Scientific American, 1084, 253, 54-65.

(14) Ramón y Cajal, S., Recuerdos de mi vida. Historia de mi labor científica, Madrid, Alianza Editorial S.A., 1981.

(15) Shepherd, G., Foundations of the neuron doctrine, New York, Oxfod Univ. Press, 1991.

(16) Swanson, L. W. "What is the brain?", Trends in Neurosciences, 2000, 23, 519-527.

(17) Bunge, M., "La filosofía es pertinente a la investigación científica del problema mente-cerebro". En: Mora, F. (Ed.), El Problema Cerebro-Mente, Alianza Editorial S.A., 1995.

(18) Kandel, E. R., Schwartz, J. H., y Jessell, T. M., Principios de Neurociencia, Madrid, McGraw-Hill / Interamericana, 2000.

(19) Edelman, G. M., Bright Air, Brilliant Fire. On the Matter of the Mind, London, Penguin Books, 1992.

(20) Rivière, A., “Mentes, cerebros y cómputos: ¿Problemas o misterios?”. En: Mora, F. (Ed.), El Problema Cerebro-Mente, Madrid, Alianza Editorial S.A. 1995.

(21) Gabbard, C. O., "Psychotherapies". En: Sadock, B. J. y Sadock, V. A. (Eds.), Kaplan \& Sadock's Comprehensive Textbook of Psychiatry, Philadelphia, Lippincott Williams \& Wilkins, 2000.

(22) Kandel, E. R. "A new intellectual framework for psychiatry", American Journal of Psychiatry, 1998, 155, 457-469.

(23) Kandel, E. R. "Biology and the future of psychoanalysis: A new intellectual framework for psychiatry revisited", American Journal of Psychiatry, 1999, 156, 505-524.

(24) Kesselring, E. "Neuroscience and clinical practice: a personal postscript", Brain Research Reviews, 2001, 36, 285-286.

(25) Andreasen, N. C. "Editor's comment: The crisis in clinical research", American Journal of Psychiatry, 1998, 155, 455- 
(26) Le Fanu, J., The Rise and Fall of Modern Medicine, London, Abacus, 1999.

(27) Sadock, B. J. y otros, Kaplan \& Sadock's Comprehensive Textbook of Psychiatry, Philadelphia, Lippincott Williams \& Wilkins, 2000.

(28) Cowan, W. M.; Harter, D. H., y Kandel, E. R. "The emergence of modern neuroscience: Some implications for neurology and psychiatry", Annual Review of Neuroscience, 2000, 23, 343-391.

(29) Kandel, E. R. y Squire, L. R. "Neuroscience: Breaking down scientific barriers to the study of brain and mind", Science, 2000, 290, 1113-1120.

(30) Jones, E. G. "Neuroscience in the modern era", SfN Newsletter, 2000, 31, 5,10-11.

(31) Midgley, M., "Why memes?". En: Rose, H. y Rose, S. (Eds.), Alas, poor Darwin. Arguments against Evolutionary Psychology, London, Jonathan Cape , 2000.

(32) Hull, D. L. "The use and abuse of Sir Karl Popper", Biological Philosophy, 1999, 14, 481-504.

(33) Bruyn, G. W. "The future of neuroscience - An ontogeny and teleology", Journal of Neuropathology and Experimental Neurology, 1997, 56, 1073-1094.

(34) Skolimowski, H., "Problemas de racionalidad en biología". En: Ayala, F. J. y Dobzhansky, T. (Eds.), Estudios sobre la filosofia de la biología, Barcelona, Ariel, S.A. 1983.

(35) Popper, K. R., "La reducción científica y la incompletitud esencial de toda ciencia". En: Ayala, F. J. y Dobzhansky, T. (Eds.), Estudios sobre la Filosofía de la Biología, Barcelona, Ariel S.A. 1983.

(36) Churchland, P. M., The engine of reason, the seat of the soul, Cambridge, MA, The MIT Press, 1996.

(37) Searle, J. R. "Consciousness", Annual Review of Neuroscience, 2000, 23, 557-578.

(38) Nagel, T., "Consciousness and objective reality". En: Warner, R. y Szubka, T. (Eds.), The Mind-Body Problem. A Guide to Current Debate, Oxford, Blackwell , 1994.

(39) Posner, M. I. y Raichle, M. E. "Précis of Images of Mind", Behavioral and Brain Sciences, $1995,18,327-383$.

(40) Albright, T. D.; Kandel, E. R., y Posner, M. I. "Cognitive neuroscience”, Curr.Opin.Neurobiol., 2000, 10, 612-624.

(41) Mesulam, M. M. "From sensation to cognition", Brain, 1998, 121, 1013-1052.

(42) Gold, I. y Stoljar, D. "A neuron doctrine in the philosophy of neuroscience", Behavioral and Brain Sciences, 1999, 22, 809-830 .

(43) Albright, T. D.; Jessell, T. M.; Kandel, E. R., y Posner, M. I. "Neural science: A century of progress and the mysteries that remain", Cell, 2000, 100 Suppl, S1-S5.

(44) Lo, D. C. "Challenges for neuroscience in a post-genome world", Nature Neuroscience, 2001, 4 Suppl. S, 1153-1154.

(45) Valenstein, E. y Charney, D. "Are we 'blaming' brain chemistry for mental illness?. A debate", Cerebrum, 2000, 2, 87-114.

*Catedrático - Ex-Presidente de la Sociedad Española de Neurociencia

Dirección y correspondencia con el autor:

Dr. C. Avendaño - Departamento de Morfología

Facultad de Medicina - Universidad Autónoma de Madrid

c/ Arzobispo Morcillo s/n - 28029 Madrid

carlos.avendano@uam.es

Recibido: 30 - 04 - 02 